\title{
Epidermal growth factor receptor signaling pathway is frequently altered in ampullary carcinoma at protein and genetic levels
}

Kaidi Mikhitarian ${ }^{1}$, Maressa Pollen ${ }^{1}$, Zhiguo Zhao ${ }^{2}$, Yu Shyr ${ }^{2}$, Nipun B Merchant ${ }^{3}$, Alexander Parikh ${ }^{3}$, Frank Revetta ${ }^{1}$, M Kay Washington ${ }^{1}$, Cindy Vnencak-Jones ${ }^{1}$ and Chanjuan Shi ${ }^{1}$

${ }^{1}$ Department of Pathology, Microbiology, and Immunology, Vanderbilt University Medical Center, Nashville, TN, USA; ${ }^{2}$ Department of Biostatistics, Vanderbilt University Medical Center, Nashville, TN, USA and ${ }^{3}$ Department of Surgery, Surgical Oncology, Vanderbilt University Medical Center, Nashville, TN, USA

\begin{abstract}
Our objective was to explore alteration of the epidermal growth factor receptor (EGFR) signaling pathway in ampullary carcinoma. Immunohistochemical studies were employed to evaluate expression of amphiregulin as well as expression and activation of EGFR. A lab-developed assay was used to identify mutations in the EGFR pathway genes, including KRAS, BRAF, PIK3CA, PTEN, and AKT1. A total of 52 ampullary carcinomas were identified, including 25 intestinal-type and 24 pancreatobiliary-type tumors, with the intestinal type being associated with a younger age at diagnosis $(P=0.03)$ and a better prognosis $(P<0.01)$. Expression of amphiregulin correlated with better differentiation $(P<0.01)$, but no difference was observed between two major histologic types. Expression and activation of EGFR was more commonly seen in the pancreatobiliary type $(P<0.01)$. Mutations were detected in $50 \%$ of the pancreatobiliary type and $60 \%$ of the intestinal type. KRAS was the most common gene mutated in the pancreatobiliary type $(42 \%)$ as well as the intestinal type $(52 \%)$. Other mutations detected included PIK3CA, SMAD4 and BRAF. KRAS mutations at codons 12 and 13 did not adversely affect overall survival. In conclusion, EGFR expression and activation were different between intestinal- and pancreatobiliary-type ampullary carcinoma. KRAS mutation was common in both histologic types; however, the incidence appeared to be lower in the pancreatobiliary type compared with its pancreatic counterpart, pancreatic ductal adenocarcinoma. Mutational analysis of the EGFR pathway genes may provide important insights into personalized treatment for patients with ampullary carcinoma.

Modern Pathology (2014) 27, 665-674; doi:10.1038/modpathol.2013.185; published online 1 November 2013
\end{abstract}

Keywords: ampullary carcinoma; epidermal growth factor receptor pathway; KRAS; PIK3CA

Ampullary carcinoma arises from the ampulla of Vater, a complex region composed of a flask-like structure formed by the confluence of the distal common bile duct and the main pancreatic duct, papilla of Vater (junction of duodenal and ampullary mucosa), and the duodenal surface of the ampulla. ${ }^{1}$ The incidence of ampullary carcinoma is low, accounting for $<1 \%$ of all digestive cancer. $^{2}$ Although ampullary carcinomas are often discovered earlier than other periampullary carcinomas because

Correspondence: Dr C Shi, MD, PhD, Department of Pathology, Microbiology, and Immunology, Vanderbilt University Medical Center, 1161 21st Avenue South, C-3321A MCN, Nashville, TN 37232, USA.

E-mail: chanjuan.shi@vanderbilt.edu

Received 15 April 2013; revised 10 August 2013; accepted 13 August 2013; published online 1 November 2013 of their unique location, $\sim 50 \%$ of patients are diagnosed at an advanced, unresectable stage. ${ }^{2}$ Surgery remains the only curative treatment for these patients. However, local recurrence and/or distal metastasis continue to be a significant problem, resulting in a 5-year survival rate of $<50 \%$. Currently, there are no effective therapies for those with an unresectable cancer or for those with recurrence or distal metastasis, and the overall prognosis for patients with ampullary carcinoma remains poor.

The ampulla of Vater contains two types of epithelia: pancreatobiliary and intestinal epithelia. Kimura et $\mathrm{al}^{3}$ first classified carcinomas arising from the ampulla of Vater into pancreatobiliary and intestinal histologic types. It has been shown that these two histologic types are associated with different premalignant lesions, cell type-specific markers, and oncogene expression. The intestinal 
type frequently begins with a tubular/villous adenoma, has a morphology identical to its colonic counterpart, and is predominantly cytokeratin (CK)7 - / CK20 + /MUC2 + /MUC5AC - / CDX2 +, whereas most pancreatobiliary type starts with flat or micropapillary dysplasia, has a morphology similar to pancreatic ductal adenocarcinoma, and is CK7 + /CK20 - /MUC2 - /MUC5AC + / CDX2 - . ${ }^{4-10}$ This histologic differentiation is thought to be one of the most important prognostic factors for ampullary carcinoma, with the intestinal type showing a much better prognosis than the pancreatobiliary type. ${ }^{4,11,12}$ However, the molecular genetic basis for the histologic difference of ampullary carcinoma remains unclear.

Activation of epidermal growth factor receptor (EGFR) has been implicated in tumorigenesis of many solid tumors including colonic adenocarcinoma and pancreatic ductal adenocarcinoma. ${ }^{13-18}$ In colonic adenocarcinoma and pancreatic ductal adenocarcinoma, amphiregulin appears to be a major ligand for EGFR-mediated activation of downstream oncogenic signaling including the KRAS/BRAF/MARP kinase, the PI3K/Akt/mTOR, and the JAK/STAT signaling pathways that contribute to tumor growth and progression. ${ }^{18-22}$ Genes in the EGFR downstream signaling pathways are frequently mutated in colon cancer and pancreatic ductal adenocarcinoma. ${ }^{23-28}$ In addition, mutations of SMAD4, also called deletion in pancreatic cancer 4 (DPC4), are detected in both diseases. ${ }^{29-32}$

In the present study, we examined the expression of amphiregulin, EGFR, and phosphor-EGFR (pEGFR) in ampullary carcinoma by immunohistochemical studies. Mutation status of several genes involved in the EGFR downstream signaling pathways and SMAD4 was also investigated using a labdeveloped multiplex PCR, multiplex single base extension assay, and capillary electrophoresis. The expressions and gene mutations were analyzed and compared between the two major ampullary cancer histologic types.

\section{Materials and methods}

\section{Patient Selection}

Between 1 January 1994 and 31 January 2011, 84 patients who underwent biopsy and/or radical resection for ampullary adenocarcinoma were identified from our pathology databases. Histological blocks were available and sufficient for immunohistochemical studies and mutational analyses in 54 subjects, including 51 with resected specimen and 3 with biopsy specimen only. One patient did not undergo resection because of multiple comorbidities. Of the 53 patients who underwent pancreatoduodenectomy (Whipple procedure), 1 died perioperatively. Both cases were excluded from the study. Patient demographics and clinical data were collected from the electronic medical record. Pathology reports and histological slides were reviewed for tumor location, size, differentiation, extension, margin status, presence or absence of perineural and lymphovascular invasion, and presence or absence of lymph node metastasis by two pathologists (KM and CS). Some of the pathologic features were not assessed in the two cases with no resection specimen available. The pathologic slides were also reviewed for pathologic types including: the intestinal type composed of intestinal-type cancer cells, the pancreatobiliary type composed of pancreatobiliary-type cancer cells, adenosquamous carcinoma, signet ring cell carcinoma, and undifferentiated carcinoma. Long-term survival status was determined by review of the medical records and through use of the social security death index. This study was approved by our Institutional Review Board.

\section{Immunohistochemistry}

Unstained sections $(4 \mu \mathrm{m})$ from formalin-fixed, paraffin-embedded tissue were first deparaffinized by routine methods. For antigen retrieval, the sections were heated to $105{ }^{\circ} \mathrm{C}$ for $20 \mathrm{~min}$ in a pH 6.0 citrate buffer for MUC2 ( Abcam, Cambridge, MA, USA; dilution: 1:300), CDX2 (Cell Signaling, Boston, MA, USA; dilution: 1:400 ), and amphiregulin (Lab Vision, Kalamazoo, MI, USA; dilution: 1:100), or to $98^{\circ} \mathrm{C}$ for $20 \mathrm{~min}$ in a pH 9.0 EDTA buffer for pEGFR (Cell Signaling; dilution: 1:150), and then allowed to cool to room temperature. Antigen retrieval was not performed for EGFR (Dako, Carpinteria, CA, USA; dilution: 1:120) labeling, but the sections were pretreated with proteinase for $5 \mathrm{~min}$. After the retrievals or the pretreatment, the tissue sections were quenched with $3 \% \mathrm{H}_{2} \mathrm{O}_{2}$ in sodium azide for $5 \mathrm{~min}$ at room temperature. Primary antibodies including anti-amphiregulin, anti-EGFR, and anti-pEGFR were then incubated with the tissue sections, followed by antibody localization using the Dako Envision ${ }^{+}$HRP-labeled polymer (Dako). Staining was visualized by $5 \mathrm{~min}$ of incubation with diaminobenzidine.

Immunohistochemical stains for MUC2 (membrane labeling), CDX2 (nuclear labeling), EGFR (membrane labeling), and pEGFR (membrane and cytoplasmic labeling) were considered positive when $>5 \%$ of the cancer cells are labeled. For amphiregulin (cytoplasmic labeling), the immunohistochemical results were scored by multiplying the staining intensity by the proportion of positive cancer cells. The intensity of stain was scaled as: 0 (negative), 1 (weak), 2 (moderate), and 3 (strong), whereas the proportion of positive cells was graded as: $0(<5 \%$ positive cells), $1(5-25 \%), 2(25-50 \%), 3$ $(50-75 \%)$, and $4(75-100 \%)$. The maximal immunohistochemical score was 12. Immunohistochemical stains were reviewed by two pathologists (KM and CS). 


\section{DNA Extraction}

DNA was extracted from 3 to $510 \mu \mathrm{m}$ sections of formalin-fixed, paraffin-embedded tissue. In all cases, tissue sections were mounted on slides for macrodissection of tumor-enriched areas to increase the percentage of tumor burden in the extracted DNA specimen to at least $20 \%$. Following cell lysis and proteinase $\mathrm{K}$ treatment, the DNA was eluted using QIAquick spin columns (Qiagen, Valencia, CA, USA).

\section{Gene Mutational Analysis}

Tumor tissue-derived DNA from 52 ampullary adenocarcinomas was screened for 62 mutations in 7 cancer genes, including AKT1 (codon: E17), BRAF (codons: G466, G469, D594, G596, V600), KRAS (codons: G12, G13, Q61, A146, K117), NRAS (codons: G12, Q61), PIK3CA (codons: H1047, E542, E545, Q546, D549), PTEN (codons: R233, R159, R267), and SMAD4 (codons: E330, D351, D355, R361), using a lab-developed assay. In general, the assay includes a multiplex PCR with 100-200 ng of template DNA, a multiplex base extension assay including the SNaPshot reaction mixture ((Life Technologies, Carlsbad, CA, USA) and mutation detection by capillary electrophoresis on an ABI Genetic Analyzer $3130 \mathrm{Xl}^{33}$ The assay has a limit of detection at $\sim 10 \%$.

\section{Statistical Analysis}

The primary end point, overall survival, was defined as the time from surgery to the date of allcause death or last follow-up. For patients' demographic and clinical variables, continuous variables were summarized using the median with the 25th and 75th percentiles (interquartile range), and frequency with percentages for categorical variables. The Wilcoxon rank-sum test was used for continuous variables, and Fisher's exact test was used for categorical variables to compare the differences between any two groups. The Kaplan-Meier method and Log-rank test were used in univariate analysis on survival outcome. Cox proportional hazard models were used in multivariable analyses to investigate the associations between the risk factors and overall survival while adjusting $a$ priori selected covariates age and tumor stage. All statistical inferences were assessed at a two-sided 5\% significant level and all summary statistics, graphics, and survival models were generated using $\mathrm{R}$ version 2.15 statistical software. ${ }^{34}$

\section{Results}

\section{Overall Clinicopathological Features}

The 52 patients included 19 females (37\%) and 33 males $(63 \%)$ with a median age of 63 years (interquartile range: 52-72 years). Resection specimens were available for analysis in 50 cases. The median size of the resected invasive carcinomas was $2.0 \mathrm{~cm}$, ranging from 0.2 to $7.0 \mathrm{~cm}$. The invasive carcinomas in all cases $(n=52)$ were graded using a four grading scale of well differentiated $(n=7,13 \%)$, moderately differentiated $(n=29,56 \%)$, poorly differentiated $(n=15,29 \%)$, and undifferentiated carcinoma $(n=1$, $2 \%$ ). In the case with undifferentiated carcinoma, very focal glandular differentiation was also observed. Lymphovascular and perineural invasion were observed in $19(38 \%)$ and $14(28 \%)$ of the 50 resected specimens, respectively, with 7 cases (14\%) showing both lymphovascular and perineural invasion. Based on the American Joint Committee on Cancer 7th edition staging system for ampullary adenocarcinoma, the 50 resected cases comprised 5 stage IA, 8 stage IB, 8 stage IIA, 28 stage IIB, and 1 stage III. Margin status was able to be assessed in 47 cases, with 44 (94\%) showing negative resection margins (R0 resection), and $3(6 \%)$ having a microscopically positive superior mesenteric artery margin (R1 resection) (Table 1).

\section{Histologic Types}

Several histologic types were identified, with intestinal $(25 / 52,48 \%$, Figure 1a) and pancreatobiliary types $(24 / 52,46 \%$, Figure 1 b) comprising the vast majority of the cases. All intestinal-type adenocarcinomas were positive for CDX2 expression with $(n=19)$ or without $(n=6)$ MUC2 expression. Three mucinous adenocarcinomas expressed both MUC2 and CDX2, and were included in the intestinal group. Two intestinal-type carcinomas harbored focal signet ring cell feature. Eighteen pancreatobiliary-type adenocarcinomas were MUC2 - /CDX2 - , 5 MUC2 - /CDX2 + (weak and focal), and 1 MUC2 + (focal)/CDX2 - . The six cases with either CDX2 or MUC2 expression had a typical morphology of pancreatic ductal adenocarcinoma. Although most of the pancreatobiliary-type tumors had a morphology of tubular (conventional) pancreatic ducal adenocarcinoma, a subset $(7 / 25,28 \%)$ were featured by complex branching papillae and secondary micropapillae, composed of cuboidal pancreatobiliary-type cells (Figure 1c). According to the 2010 WHO classification of tumors of the digestive system, the latter was classified as invasive papillary adenocarcinoma, pancreatobiliary type. Other minor subtypes included 1 signet ring cell carcinoma, 1 adenosquamous carcinoma, and 1 undifferentiated carcinoma.

Patients with a pancreatobiliary-type carcinoma were older than those with an intestinal-type carcinoma (median 60, interquartile range 50-65, $n=25$ vs $69,62-74, n=24 ; P=0.028$ ). However, there was no significant gender difference $(P=0.377)$, with males outnumbering females in both groups (Table 2). Pancreatobiliary-type carcinomas tended to be diagnosed at a more advanced stage than intestinal-type carcinomas; $46 \%(11 / 24)$ 
Table 1 Overall patient demographics and clinicopathologic features

\begin{tabular}{lr}
\hline & All cases $(\mathrm{N}=52)^{\mathrm{a}}$ \\
\hline Age (years) & $63(52-72)$ \\
Sex & \\
Male & $33(63)$ \\
Female & $19(37)$ \\
& \\
Differentiation & \\
Well & $7(13)$ \\
Moderately & $29(56)$ \\
Poorly & $15(29)$ \\
Undifferentiated & $1(2)$ \\
Invasive tumor size (cm, $n=50)$ & $(1.2-3.2)$ \\
Tumor stage (n=50) & \\
Stage IA & $5(10)$ \\
Stage IB & $8(16)$ \\
Stage IIA & $8(16)$ \\
Stage IIB & $28(56)$ \\
Stage III & $1(2)$ \\
LVI and PNI (n=50) & \\
LVI only & $12(24)$ \\
PNI only & $7(14)$ \\
LVI + PNI & $7(14)$ \\
Neither LVI nor PNI & $24(48)$ \\
Resection margin status (n=47) & \\
Positive & \\
Negative & $3(6)$ \\
\hline
\end{tabular}

LVI, lymphovascular invasion; PNI, perineural invasion.

${ }^{a}$ Median (interquartile range) for continuous variables and $N(\%)$ for categorical variables.

of the patients with an intestinal-type cancer were diagnosed with a stage I tumor, whereas majority of the patients $(21 / 23,91 \%)$ with a pancreatobiliarytype cancer had either a stage II or stage III tumor $(P=0.008$, Table 2). Median overall survival from date of diagnosis was 110 months (73 to NA) and 29 months (16 to NA) for patients with an intestinaltype and a pancreatobiliary-type carcinoma, respectively. Univariate analysis showed that the patients with a pancreatobiliary-type cancer had a worse overall survival than those with an intestinal-type cancer ( $P=0.013$, Figure 2a). When the patients' age and tumor stage were adjusted, compared with those with an intestinal-type cancer, patients with a pancreatobiliary-type cancer had 2.15 times higher hazard (95\% CI: 0.75-6.16), although this difference did not reach statistical significance $(P=0.153)$.

\section{Expression of Amphiregulin and EGFR in Ampullary Adenocarcinoma}

Amphiregulin, a major ligand for EGFR, was highly expressed in most (43 of 52, 82.7\%) of the ampullary adenocarcinomas (Figures $3 \mathrm{a}$ and $\mathrm{b}$ ). There was no difference in amphiregulin expression between the pancreatobiliary and intestinal groups $(P=0.696)$. However, strong and diffuse amphiregulin labeling was more frequently observed in invasive papillary
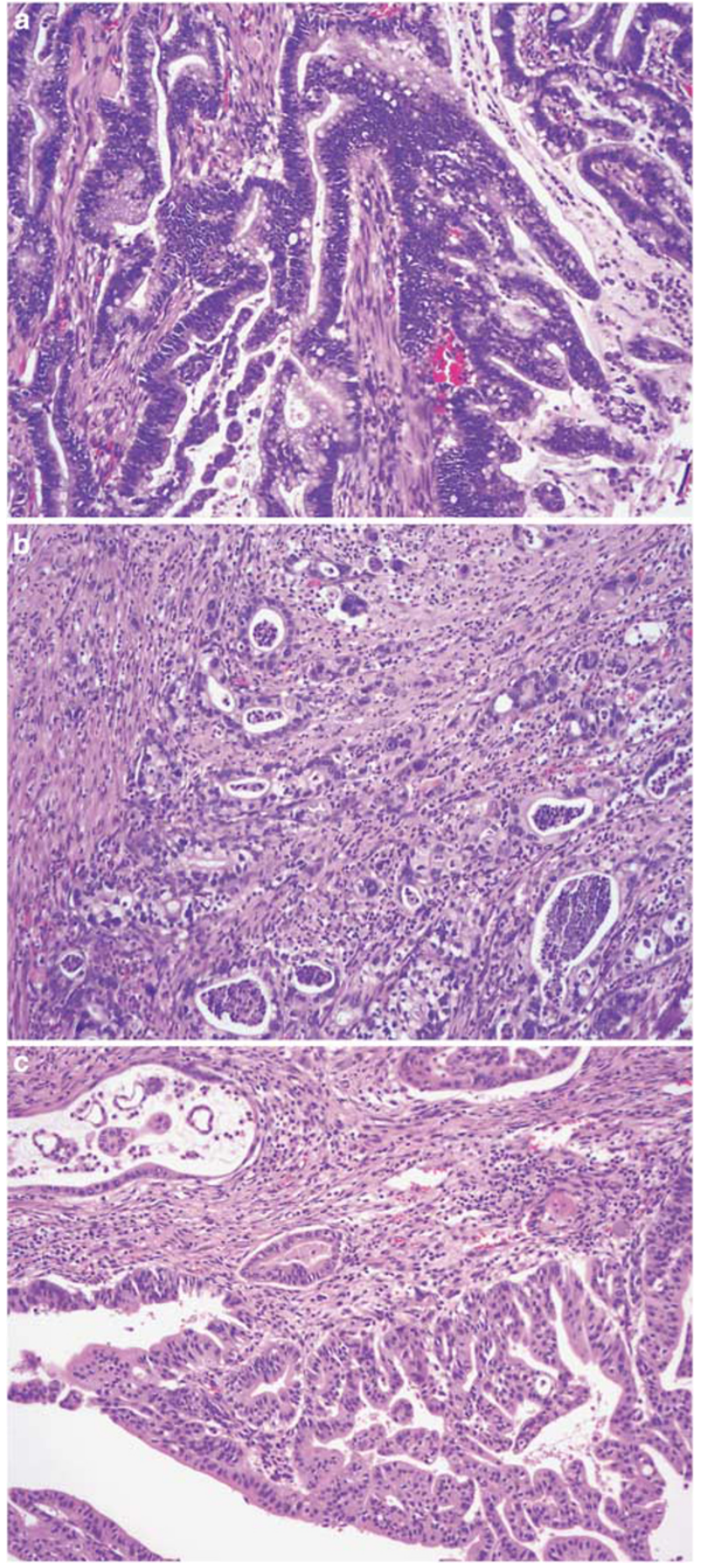

Figure 1 Major histologic types of ampullary carcinoma. (a) Intestinal type (hematoxylin and eosin, original magnification $\times 200$ ); (b) pancreatobiliary type with conventional pancreatic ductal adenocarcinoma morphology (hematoxylin and eosin, original magnification $\times 100$ ); $(\mathbf{c})$ invasive papillary adenocarcinoma, pancreatobiliary type (hematoxylin and eosin, original magnification $\times 100$ ).

adenocarcinoma, pancreatobiliary type (Figure $3 \mathrm{~b}$ ) than in all other cases $(6 / 7,86 \%$ vs $8 / 45,18 \%$; $P<0.001)$. In addition, well and moderately differentiated carcinomas (median 8.5, interquartile range 
Table 2 Patient demographics, tumor stage, alterations in the EGFR signaling pathway, and survival in patients with pancreatobiliary- and intestinal-type ampullary adenocarcinomas

\begin{tabular}{|c|c|c|c|}
\hline & $\begin{array}{l}\text { Intestinal } \\
(\mathrm{N}=25)^{\mathrm{a}}\end{array}$ & $\begin{array}{l}\text { Pancreatobiliary } \\
\qquad(\mathrm{N}=24)^{\mathrm{a}}\end{array}$ & $\mathrm{P}$-value \\
\hline Age (years) & $60(50-65)$ & $69(62-74)$ & $0.028^{\mathrm{b}}$ \\
\hline Sex & & & $0.377^{\mathrm{C}}$ \\
\hline Female & $44 \%(11)$ & $29 \%(7)$ & \\
\hline Male & $56 \%(14)$ & $71 \%(17)$ & \\
\hline Tumor stage & & & $0.008^{\mathrm{C}}$ \\
\hline $\mathrm{I}$ & $46 \%(11)$ & $9 \%(2)$ & \\
\hline II-III & $54 \%$ & $91 \%(21)$ & \\
\hline $\begin{array}{l}\text { AR immunohisto- } \\
\text { chemistry score }\end{array}$ & $8(3-12)$ & $5(2-12)$ & $0.696^{\mathrm{b}}$ \\
\hline EGFR & & & $0.002^{\mathrm{C}}$ \\
\hline Positive & $4 \%(1)$ & $42 \%(10)$ & \\
\hline Negative & $96 \%(24)$ & $58 \%(14)$ & \\
\hline$p E G F R$ & & & $0.002^{\mathrm{C}}$ \\
\hline Positive & $0 \%(0)$ & $33 \%(8)$ & \\
\hline Negative & $100 \%(25)$ & $67 \%(16)$ & \\
\hline Gene mutation & & & $0.223^{\mathrm{C}}$ \\
\hline $\begin{array}{l}\text { KRAS codons } 12 \\
\text { and } 13\end{array}$ & $52 \%(13)$ & $29 \%(7)$ & \\
\hline Other mutation & $8 \%(2)$ & $21 \%(5)$ & \\
\hline No mutation & $40 \%(10)$ & $50 \%(12)$ & \\
\hline $\begin{array}{l}\text { Overall survival } \\
\text { status }\end{array}$ & & & $0.021^{\mathrm{C}}$ \\
\hline Alive & $60 \%$ & $25 \%(6)$ & \\
\hline Dead & $40 \%(10)$ & $75 \%(18)$ & \\
\hline $\begin{array}{l}\text { Follow-up time } \\
\text { (months) }\end{array}$ & $52(29-83)$ & 27 (14-69) & $0.093^{\mathrm{b}}$ \\
\hline
\end{tabular}

EGFR, epidermal growth factor receptor; AR, amphiregulin; pEGFR, phosphor-EGFR.

${ }^{\mathrm{a}}$ Median (interquartile range) for continuous variables and $N(\%)$ for categorical variables.

bilcoxon test.

${ }^{\mathrm{C}}$ Fisher's exact test.

4.0-12.0, $n=36$ ) were more likely to express amphiregulin compared with those with poor differentiation and undifferentiation (median 1.0, interquartile range $0-4.5, \quad n=16, P<0.001)$. All the seven invasive papillary adenocarcinomas were moderately differentiated. The signet ring cell carcinoma, adenosquamous carcinoma, and undifferentiated carcinoma showed minimal to no amphiregulin expression.

EGFR expression was detected in 13 of $52(25 \%)$ invasive carcinomas by immunohistochemistry (Figure 3c). Ten of 24 (42\%) pancreatobiliary-type tumors were labeled with anti-EGFR antibody (Figure 3c), whereas only 1 of 25 (4\%) intestinaltype tumors expressed EGFR. EGFR was more commonly expressed in pancreatobiliary- than in intestinal-type tumors $(P=0.002)$. Activated EGFR (pEGFR) was detected in 10 of 52 cases (19\%) by immunohistochemistry (Figure 3d). Similar to EGFR expression, the activation of EGFR was more common in pancreatobiliary- than in intestinal- type tumors $(8 / 24,33 \%$ vs $0 / 25,0 \% ; P=0.002)$. Neither EGFR labeling nor activation of EGFR was observed
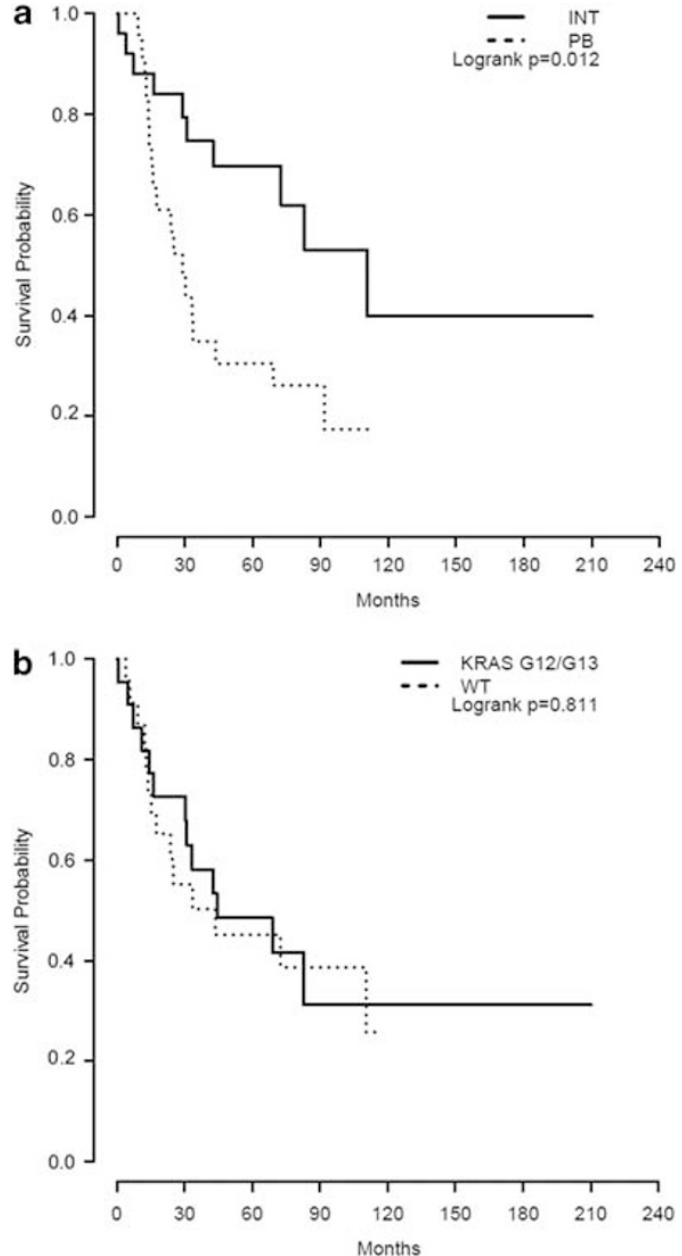

Figure 2 Effect of histologic types and KRAS mutation status on survival. (a) Kaplan-Meier survival analysis of patients with intestinal- and pancreatobiliary-type ampullary carcinoma. (b) Kaplan-Meier survival analysis of patients with a KRAS wild-type (WT) and a KRAS G12/13 mutant tumor.

in the single signet ring cell carcinoma case. Interestingly, both adenosquamous and undifferentiated carcinomas displayed expression and activation of EGFR. The activation of EGFR was negatively correlated with amphiregulin expression $(P=0.017)$.

\section{Gene Mutations in Ampullary Adenocarcinoma}

One or more mutations were detected in 29 of 52 (56\%) tumors (Table 3). As expected, KRAS was the most commonly mutated gene detected in ampullary carcinoma; 25 of 52 (48\%) tumors harbored one $(n=24)$ or two KRAS mutations $(n=1)$, including 21 $(40 \%)$ with $1(n=20)$ or $2(n=1)$ mutations at codon $12,1(2 \%)$ with a codon 13 mutation (p.G13D), and 3 $(6 \%)$ with a codon 61 mutation (p.Q61H). Interestingly, one case carried two different types of KRAS mutations, with p.G12R being the major component and p.G12D the minor component, suggesting tumor heterogeneity.

Among the 25 cases with KRAS mutation, 20 (20/ $52,38 \%$ ) had mutation only in the KRAS gene. The 

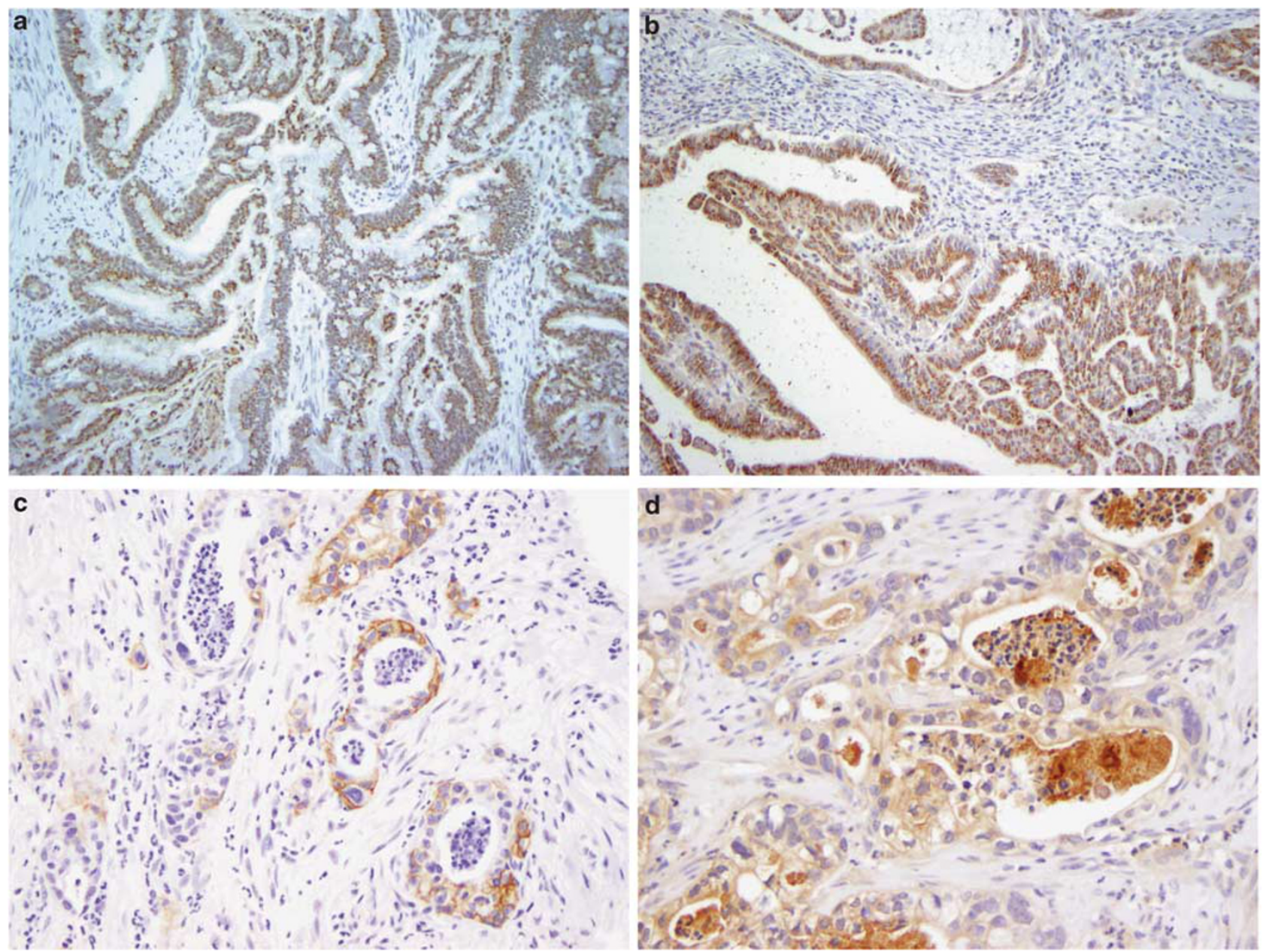

Figure 3 Expression of amphiregulin, EGFR, and phosphor-EGFR (pEGFR). (a) Immunohistochemical labeling for amphiregulin of the tumor in Figure 1a (original magnification $\times 200$ ). (b) Immunohistochemical labeling for amphiregulin of the tumor in Figure 1c (original magnification $\times 100)$. (c) Immunohistochemical labeling for EGFR of the tumor in Figure 1b (original magnification $\times 200$ ). (d) Immunohistochemical labeling for pEGFR of the tumor in Figure 1b (original magnification $\times 200$ ).

Table 3 Mutations in the EGFR pathway genes and SMAD4 in intestinal- and pancreatobiliary- type ampullary carcinomas

\begin{tabular}{|c|c|c|c|c|c|c|c|c|c|}
\hline & $\begin{array}{c}\text { KRAS } \\
\text { codon } 12\end{array}$ & $\begin{array}{c}\text { KRAS } \\
\text { codon } 13\end{array}$ & $\begin{array}{c}\text { KRAS } \\
\text { codon } 61\end{array}$ & $B R A F$ & PIKЗCA & $\begin{array}{l}\text { KRAS + } \\
\text { PIK3CA }\end{array}$ & $\begin{array}{l}\text { KRAS + } \\
\text { SMAD4 }\end{array}$ & $\begin{array}{c}\text { KRAS + } \\
\text { PIK3CA + SMAD4 }\end{array}$ & Total (\%) \\
\hline Intestinal $(n=25)$ & 8 & 0 & 0 & 0 & 2 & 2 & $2^{\mathrm{a}}$ & 1 & $15(60)$ \\
\hline Pancreatobiliary $(n=24)$ & 7 & 0 & 3 & 1 & 1 & 0 & 0 & 0 & $12(50)$ \\
\hline Signet ring cell $(n=1)$ & 1 & 0 & 0 & 0 & 0 & 0 & 0 & 0 & $1(100)$ \\
\hline Adenosquamous $(n=1)$ & 1 & 0 & 0 & 0 & 0 & 0 & 0 & 0 & $1(100)$ \\
\hline Undifferentiated $(n=1)$ & 0 & 0 & 0 & 0 & 0 & 0 & 0 & 0 & $0(0)$ \\
\hline Total & 17 & 0 & 3 & 1 & 3 & 2 & 2 & 1 & 29 \\
\hline
\end{tabular}

EGFR, epidermal growth factor receptor.

${ }^{a}$ One of KRAS mutation was in codon 13.

other $5(5 / 52,10 \%)$ tumors contained coexisting mutation(s) in one or two other genes: 2 with a PIK3CA exon 9 mutation, 2 with a SMAD4 mutation, and 1 with both SMAD4 and PIK3CA exon 9 mutation. In the case with three gene mutations, KRAS p.G12C mutant cells accounted for the majority of the cells tested, whereas only $\sim 20 \%$ of the cells harbored PIK3CA and SMAD4 mutations (Figure 4).

Of the 52 cases, $6(12 \%)$ had a mutation in PIK3CA, all in exon 9 (5 p.E545K and 1 p.Q546R).
Three of them had coexisting mutations in other genes: 2 KRAS and 1 KRAS + SMAD4. Although PIK3CA can be the only EGFR pathway gene mutated in ampullary carcinoma, SMAD4 mutation seemed to be only detected in the tumors with one or more mutations in other genes. Of the 52 cases, 3 $(6 \%)$ harbored a $S M A D 4$ mutation, all of which had coexisting mutations in KRAS and/or PIK3CA.

Multiple BRAF mutations were analyzed; however, p.V600E is the only mutation detected, and 

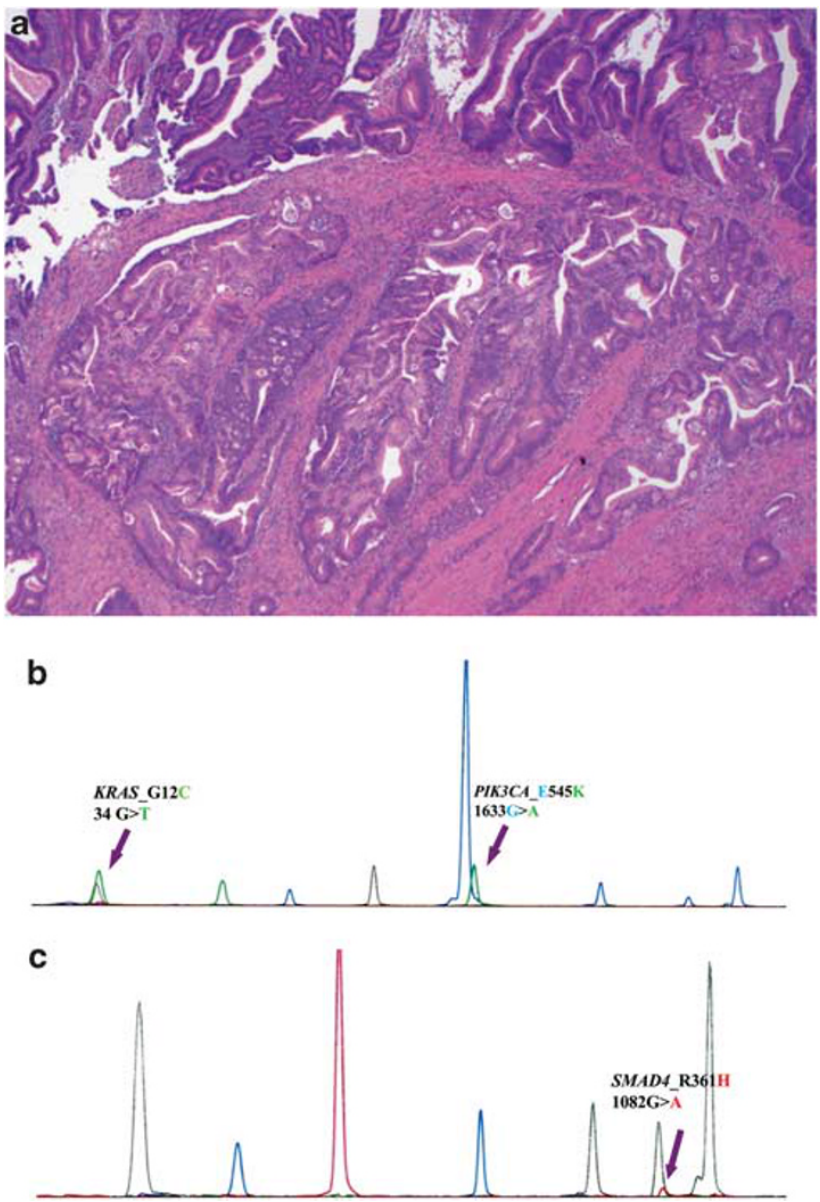

Figure 4 An intestinal-type ampullary carcinoma with multiple gene mutations. (a). Hematoxylin and eosin section of the tumor showing invasive carcinoma arising in a tubular adenoma (hematoxylin and eosin stain, original magnification $\times 100$ ). (b) A panel showing mutations in KRAS and PIK3CA. (c) Another panel showing a mutation in SMAD4.

only one case contained the mutation. Curiously, this mutation was detected in one invasive papillary adenocarcinoma, pancreatobiliary type. Unexpectedly, no intestinal-type tumors $(0 / 25,0 \%)$ had $B R A F$ mutation identified. No mutation was detected in NRAS, PTEN, and AKT1.

Twelve of $24(50 \%)$ pancreatobiliary-type tumors harbored one mutation in the genes tested, including $7(7 / 24,29 \%)$ in KRAS codon $12,3(3 / 24,13 \%)$ in KRAS codon 61, $1(1 / 24,4 \%)$ in PIK3CA codon 545, and $1(1 / 24,4 \%)$ in BRAF codon 600. Among the 7 pancreatobiliary-type invasive papillary adenocarcinomas, only $2(29 \%)$ contained either a KRAS or a BRAF mutation. On the other hand, 10 of 17 (59\%) pancreatobiliary-type tumors with conventional ductal adenocarcinoma morphology had either KRAS or PIK3CA mutation. However, statistically there was no difference in the mutation frequency between the two morphologies $(P=0.37)$.

Fifteen of $25(60 \%)$ intestinal-type tumors had one or more mutations. These included $8(32 \%)$ with 1 $(n=7)$ or 2 mutations $(n=1)$ in KRAS codon 12 only,
$5(20 \%)$ with a mutation in KRAS codon 12 or 13 plus a mutation in PIK3CA and/or SMAD4, and $2(8 \%)$ with a mutation in PIK3CA codon 545 only. KRAS mutation was seen in 13 of 25 (52\%) intestinal-type vs 10 of 24 (42\%) pancreatobiliary-type tumors. There was no significant difference in KRAS mutation status between the two groups $(P=0.57)$.

Mutations in KRAS codons 12 and 13 have been reported to be a prognostic factor for colonic adenocarcinoma, with the mutations being associated with a poor prognosis. The effect of KRAS codon 12 and 13 mutations on overall survival of the patients with ampullary carcinoma was assessed using Kaplan-Meier analysis, which demonstrated no association with overall survival (Figure 2b). Median (95\% CI) for overall survival from date of diagnosis was 45 months ( 31 to NA) and 44 months (18 to NA) for patients with a tumor with the mutations and for those with a tumor without the mutations $(P=0.811)$.

\section{Discussion}

Ampullary carcinomas with intestinal and pancreatobiliary differentiation comprise the vast majority of carcinomas arising in the ampulla of Vater. Although some studies demonstrated that intestinal-type ampullary carcinomas were associated with a better prognosis compared with the pancreatobiliary type, ${ }^{11,12}$ others reported that stage by stage, they tended to have the same prognosis. ${ }^{7,25,35}$ Our results were largely consistent with both findings; univariate analysis showed that patients with a pancreatobiliary-type cancer had a worse overall survival compared with those with an intestinal-type carcinoma, whereas the difference in overall survival became not so significant when the patients' age and tumor stage were adjusted. Interestingly, our study showed that patients diagnosed with a pancreatobiliary-type carcinoma were older than those with an intestinal-type carcinoma. Older age may partly confer a poorer survival in patients with a pancreatobiliary-type tumor.

Based on the location, Adsay et $a l^{1}$ recently classified ampullary carcinomas into four different subtypes: (1) intra-ampullary carcinoma arising in intra-ampullary papillary-tubular neoplasms; (2) ampullary-ductal infiltrative carcinomas that are presumably due to the pancreatobiliary histology/ origin; (3) peri-ampullary-duodenal carcinoma with significant adenoma component; and (4) ampullary carcinoma-not otherwise specified arising at the papilla of Vater. According to the above classification, the majority of ampullary adenocarcinomas in this series were either ampullary-ductal infiltrative carcinomas or ampullary carcinomasnot otherwise specified. The seven invasive papillary adenocarcinomas may be classified as intra-ampullary carcinomas. Intra-ampullary carcinomas were associated with a lower TMN stage, and 
were more likely to be intestinal type in the study of Adsay et $a l ;{ }^{1}$ however, six of the seven cases in our series were T3N1M0, and all of them were pancreatobiliary type.

EGFR ligands are overexpressed in a majority of colonic adenocarcinomas, with amphiregulin being the EGFR ligand with the most enhanced expression. ${ }^{36}$ Amphiregulin has been shown to stimulate EGFR activation and tumor cell growth in colon cancer cell lines. ${ }^{20}$ In addition, amphiregulin expression may be related to disease-free survival and liver metastasis. ${ }^{36-40}$ In pancreatic ductal adenocarcinoma, amphiregulin has been demonstrated to be involved in the tumor progression through the activation of EGFR. ${ }^{19,41}$ Amphiregulin is also a potential target for pancreatic cancer therapy. ${ }^{19,42}$ In addition, negative expression of amphiregulin was reported to be associated with an unfavorable prognosis in pancreatic cancer. ${ }^{43}$ The present study showed that amphiregulin was frequently expressed by both pancreatobiliary- and intestinal-type ampullary carcinomas. Expression of amphiregulin was associated with better differentiation. However, no difference in amphiregulin expression was observed in two major histologic types. Other variants of ampullary carcinomas including adenosquamous carcinoma, signet ring cell carcinoma, and undifferentiated carcinoma displayed none to little amphiregulin expression.

Enhanced EGFR expression is also observed in both colorectal cancer and pancreatic ductal adenocarcinoma. ${ }^{13-18}$ EGFR overexpression and activation were detected in up to $50 \%$ of colonic adenocarcinomas. ${ }^{44,45}$ However, the expression and activation of EGFR may be infrequent in intestinaltype ampullary carcinomas, as only one intestinaltype tumor expressed EGFR and none of them had activation of EGFR detected by immunohistochemistry. On the other hand, a significant portion of pancreatobiliary-type ampullary carcinomas had expression and/or activation of EGFR detected by immunohistochemistry. These results suggest that EGFR may play different roles in carcinogenesis of the two histologic types. In addition, our results demonstrated that positive pEGFR expression was associated with lower amphiregulin expression, indicating that amphiregulin may not be a major contributor for activation of EGFR in ampullary adenocarcinoma.

Frequencies of KRAS, BRAF, and PIK3CA mutations have been described in carcinomas of pancreatobiliary origin. The reported prevalence of KRAS mutation in extrahepatic cholangiocarcinomas ranges from 0 to $100 \%$, whereas $B R A F$ and PI3KCA mutations have been consistently rare in all studies. ${ }^{46-49}$ One study specifically looked into mutational spectrum of 13 carcinomas of the distal common bile duct and found no mutations in these three genes. ${ }^{48}$ Like extrahepatic cholangiocarcinoma, mutations in BRAF and PIK3CA are also infrequent in pancreatic ductal adenocarcinoma; however, KRAS is almost universally mutated in this cancer. ${ }^{50-52}$ A few studies have investigated mutations of KRAS and BRAF in ampullary carcinoma. Although $20-67 \%$ of ampullary carcinomas harbor KRAS mutation, only up to $10 \%$ have a mutation in $B R A F^{4,53,54}$ Only one study examined PIK3CA mutation in ampullary carcinomas and reported no PIK3CA mutations in 21 cases. ${ }^{54}$ Consistent to these studies, we observed $\sim 50 \%$ of ampullary carcinomas with one or two KRAS mutations and only one case $(2 \%)$ with a $B R A F$ mutation. In addition, we also detected PIK3CA mutation in $10 \%$ of the cases.

As described above, activating mutations in the KRAS proto-oncogene occur almost ubiquitously in pancreatic ductal adenocarcinoma, ${ }^{51}$ with the vast majority of the mutations occurring at codon 12 . On the contrary, we only observed KRAS mutations in 10 of $24(42 \%)$ pancreatobiliary-type carcinomas: 7 $(29 \%)$ at codon 12 and $3(13 \%)$ at codon 61 . Mutation at KRAS codon 61 is a rare event in pancreatic ductal adenocarcinoma. These data suggest that KRAS may play different roles in carcinogenesis of pancreatobiliary-type ampullary carcinoma and pancreatic ductal adenocarcinoma. On the other hand, our data showed that pancreatobiliary-type ampullary carcinoma frequently expressed EGFR and in some cases activated EGFR was detected by immunohistochemistry. Therefore, it is rational to postulate that anti-EGFR therapy may be effective at least for some pancreatobiliary-type ampullary carcinomas, in contrast to pancreatic ductal adenocarcinoma, where essentially no response is observed because of an almost universal KRAS mutation. Therefore, KRAS mutational analysis of ampullary carcinoma could guide treatment of patients with advanced-stage ampullary carcinoma.

In the pancreatobiliary histologic type, invasive papillary adenocarcinoma variant showed a unique histologic morphology. ${ }^{55}$ The tumors were composed of cuboidal pancreatobiliary-type cancer cells, and therefore were classified as pancreatobiliary type. Rather than forming tubular structures seen in conventional pancreatic ductal adenocarcinoma, the cancer cells formed a complex architecture with prominent secondary micropapillae. The subgroup was strongly associated with amphiregulin overexpression, with six of seven cases being intensely and diffusely labeled with anti-amphiregulin antibody. KRAS mutation was only detected in 1 of $7(15 \%)$ of these cases, and 1 tumor harbored a BRAF V600E mutation. It appeared that the molecular mechanism underlying this subgroup is different from that of ampullary adenocarcinoma with a morphology similar to pancreatic ductal adenocarcinoma; however, the sample size is small, and therefore a definitive difference cannot be determined.

Approximately $50 \%$ intestinal-type ampullary carcinomas had a mutation in the KRAS gene, which is compatible with colonic adenocarcinoma. Similar to colonic adenocarcinoma, a PI3KCA 
mutation was detected in $\sim 20 \%$ of intestinal-type ampullary carcinoma, with some of the mutations coexisting with a KRAS and/or a SMAD4 mutation. ${ }^{23,24,27}$ BRAF mutation is detected in $10-15 \%$ of colonic adenocarcinomas. ${ }^{23,56-58}$ In our series, none of 25 intestinal-type cancers harbored a mutation in the $B R A F$ gene. It is well known that $B R A F$ gene mutation is more frequently seen in colon cancers that follow a serrated pathway. Our data suggest that the serrated pathway is rarely involved in the carcinogenesis of ampullary carcinoma.

In conclusion, alterations in the EGFR signaling pathway are frequently present in ampullary carcinoma. KRAS mutation is common; however, compared with conventional pancreatic ductal adenocarcinoma, the incidence of KRAS mutation is lower in pancreatobiliary-type ampullary carcinoma. Therefore, anti-EGFR therapy might be effective in some ampullary carcinomas, including both histologic types. Mutational analysis of the EGFR pathway genes in ampullary carcinoma may provide valuable information for cancer treatment.

\section{Acknowledgments}

This project is funded by NIH/NCI P50CA095103 (to KW and CS).

\section{Disclosure/conflict of interest}

The authors declare no conflict of interest.

\section{References}

1 Adsay V, Ohike N, Tajiri T, et al. Ampullary region carcinomas: definition and site specific classification with delineation of four clinicopathologically and prognostically distinct subsets in an analysis of 249 cases. Am J Surg Pathol 2012;36:592-1608.

2 Benhamiche AM, Jouve JL, Manfredi S, et al. Cancer of the ampulla of Vater: results of a 20-year populationbased study. Eur J Gastroenterol Hepatol 2000;12: 75-79.

3 Kimura W, Futakawa N, Yamagata S, et al. Different clinicopathologic findings in two histologic types of carcinoma of papilla of Vater. Jpn J Cancer Res 1994;85: 161-166.

4 Kohler I, Jacob D, Budzies J, et al. Phenotypic and genotypic characterization of carcinomas of the papilla of Vater has prognostic and putative therapeutic implications. Am J Clin Pathol 2011;135:202-211.

5 Chu PG, Schwarz RE, Lau SK, et al. Immunohistochemical staining in the diagnosis of pancreatobiliary and ampulla of Vater adenocarcinoma: application of CDX2, CK17, MUC1, and MUC2. Am J Surg Pathol 2005;29:359-367.

6 Baumhoer D, Zlobec I, Tornillo L, et al. Immunophenotyping and oncogene amplifications in tumors of the papilla of Vater. Virchows Arch 2008;453:579-588.

7 Zhou H, Schaefer N, Wolff M, et al. Carcinoma of the ampulla of Vater: comparative histologic/immuno- histochemical classification and follow-up. Am J Surg Pathol 2004;28:875-882.

8 Matsubayashi H, Watanabe H, Ajioka Y, et al. Different amounts of K-ras mutant epithelial cells in pancreatic carcinoma and mass-forming pancreatitis. Pancreas 2000;21:77-85.

9 Sessa F, Furlan D, Zampatti C, et al. Prognostic factors for ampullary adenocarcinomas: tumor stage, tumor histology, tumor location, immunohistochemistry and microsatellite instability. Virchows Arch 2007;451: 649-657.

10 Fischer HP, Zhou H. Pathogenesis of carcinoma of the papilla of Vater. J Hepatobiliary Pancreat Surg 2004; 11:301-309.

11 Kim WS, Choi DW, Choi SH, et al. Clinical significance of pathologic subtype in curatively resected ampulla of vater cancer. J Surg Oncol 2012;105:266-272.

12 Carter JT, Grenert JP, Rubenstein L, et al. Tumors of the ampulla of vater: histopathologic classification and predictors of survival. J Am Coll Surg 2008;207:210-218.

$13 \mathrm{Kim}$ ES. Epidermal growth factor receptor as a target in cancer therapy. J Natl Compr Canc Netw 2003;1 (Suppl 1):S87-S95.

14 O’Dwyer PJ, Benson AB 3rd. Epidermal growth factor receptor-targeted therapy in colorectal cancer. Semin Oncol 2002;29:10-17.

15 Cohen RB. Epidermal growth factor receptor as a therapeutic target in colorectal cancer. Clin Colorectal Cancer 2003;2:246-251.

16 Valsecchi ME, McDonald M, Brody JR, et al. Epidermal growth factor receptor and insulinlike growth factor 1 receptor expression predict poor survival in pancreatic ductal adenocarcinoma. Cancer 2012;118:3484-3493.

17 Tzeng CW, Frolov A, Frolova N, et al. EGFR genomic gain and aberrant pathway signaling in pancreatic cancer patients. J Surg Res 2007;143:20-26.

18 Higginbotham JN, Demory Beckler M, Gephart JD, et al. Amphiregulin exosomes increase cancer cell invasion. Curr Biol 2011;21:779-786.

19 Yotsumoto F, Fukami T, Yagi H, et al. Amphiregulin regulates the activation of ERK and Akt through epidermal growth factor receptor and HER3 signals involved in the progression of pancreatic cancer. Cancer Sci 2010;101:2351-2360.

20 Merchant NB, Rogers CM, Trivedi B, et al. Liganddependent activation of the epidermal growth factor receptor by secondary bile acids in polarizing colon cancer cells. Surgery 2005;138:415-421.

21 Bardelli A, Siena S. Molecular mechanisms of resistance to cetuximab and panitumumab in colorectal cancer. J Clin Oncol 2010;28:1254-1261.

22 Silva CM. Role of STATs as downstream signal transducers in Src family kinase-mediated tumorigenesis. Oncogene 2004;23:8017-8023.

23 Sun T, Simon I, Moreno V, et al. A combined oncogenic pathway signature of BRAF, KRAS and PI3KCA mutation improves colorectal cancer classification and cetuximab treatment prediction. GUT 2013;62:540-549.

24 De Roock W, Claes B, Bernasconi D, et al. Effects of KRAS, BRAF, NRAS, and PIK3CA mutations on the efficacy of cetuximab plus chemotherapy in chemotherapy-refractory metastatic colorectal cancer: a retrospective consortium analysis. Lancet Oncol 2010; 11:753-762.

25 Tol J, Dijkstra JR, Klomp M, et al. Markers for EGFR pathway activation as predictor of outcome in 
metastatic colorectal cancer patients treated with or without cetuximab. Eur J Cancer 2010;46:1997-2009.

26 Lievre A, Blons H, Laurent-Puig P. Oncogenic mutations as predictive factors in colorectal cancer. Oncogene 2010;29:3033-3043.

27 Baldus SE, Schaefer KL, Engers R, et al. Prevalence and heterogeneity of KRAS, BRAF, and PIK3CA mutations in primary colorectal adenocarcinomas and their corresponding metastases. Clin Cancer Res 2010;16:790-799.

28 Immervoll H, Hoem D, Kugarajh K, et al. Molecular analysis of the EGFR-RAS-RAF pathway in pancreatic ductal adenocarcinomas: lack of mutations in the BRAF and EGFR genes. Virchows Arch 2006;448: 788-796.

29 Moore PS, Sipos B, Orlandini S, et al. Genetic profile of 22 pancreatic carcinoma cell lines. Analysis of K-ras, p53, p16 and DPC4/Smad4. Virchows Arch 2001;439:798-802.

30 Koyama $\mathrm{M}$, Ito $\mathrm{M}$, Nagai $\mathrm{H}$, et al. Inactivation of both alleles of the DPC4/SMAD4 gene in advanced colorectal cancers: identification of seven novel somatic mutations in tumors from Japanese patients. Mutat Res 1999;406:71-77.

31 Schutte M. DPC4/SMAD4 gene alterations in human cancer, and their functional implications. Ann Oncol 1999;10(Suppl 4):56-59.

32 Woodford-Richens KL, Rowan AJ, Gorman P, et al. SMAD4 mutations in colorectal cancer probably occur before chromosomal instability, but after divergence of the microsatellite instability pathway. Proc Natl Acad Sci USA 2001;98:9719-9723.

$33 \mathrm{Su} \mathrm{Z}$, Dias-Santagata D, Duke M, et al. A platform for rapid detection of multiple oncogenic mutations with relevance to targeted therapy in non-small-cell lung cancer. J Mol Diagn 2011;13:74-84.

34 Team RDC A Language and Environment for Statistical Computing. R Foundation for Statistical Computing: Vienna, Austria, 2012.

35 de Paiva Haddad LB, Patzina RA, Penteado S, et al. Lymph node involvement and not the histophatologic subtype is correlated with outcome after resection of adenocarcinoma of the ampulla of vater. J Gastrointest Surg 2010;14:719-728.

36 Ohchi T, Akagi Y, Kinugasa T, et al. Amphiregulin is a prognostic factor in colorectal cancer. Anticancer Res 2012;32:2315-2321.

37 Yamada M, Ichikawa Y, Yamagishi S, et al. Amphiregulin is a promising prognostic marker for liver metastases of colorectal cancer. Clin Cancer Res 2008; 14:2351-2356.

$38 \mathrm{Li}$ XD, Miao SY, Wang GL, et al. Amphiregulin and epiregulin expression in colorectal carcinoma and the correlation with clinicopathological characteristics. Onkologie 2010;33:353-358.

39 Kuramochi H, Nakajima G, Kaneko Y, et al. Amphiregulin and Epiregulin mRNA expression in primary colorectal cancer and corresponding liver metastases. BMC Cancer 2012;12:88.

40 Khambata-Ford S, Garrett CR, Meropol NJ, et al. Expression of epiregulin and amphiregulin and K-ras mutation status predict disease control in metastatic colorectal cancer patients treated with cetuximab. J Clin Oncol 2007;25:3230-3237.

41 Dong A, Gupta A, Pai RK, et al. The human adenocarcinoma-associated gene, AGR2, induces expression of amphiregulin through Hippo pathway co-activator YAP1 activation. J Biol Chem 2011;286:18301-18310.

42 Yotsumoto F, Yagi H, Suzuki SO, et al. Validation of HB-EGF and amphiregulin as targets for human cancer therapy. Biochem Biophys Res Commun 2008;365: 555-561.

43 Park JK, Kim MA, Ryu JK, et al. Postoperative prognostic predictors of pancreatic ductal adenocarcinoma: clinical analysis and immunoprofile on tissue microarrays. Ann Surg Oncol 2012;19:2664-2672.

44 Rego RL, Foster NR, Smyrk TC, et al. Prognostic effect of activated EGFR expression in human colon carcinomas: comparison with EGFR status. Br J Cancer 2010;102:165-172.

45 Galizia G, Lieto E, Ferraraccio F, et al. Prognostic significance of epidermal growth factor receptor expression in colon cancer patients undergoing curative surgery. Ann Surg Oncol 2006;13:823-835.

46 Voss JS, Holtegaard LM, Kerr SE, et al. Molecular profiling of cholangiocarcinoma shows potential for targeted therapy treatment decisions. Hum Pathol 2013;44:1216-1222.

47 Hezel AF, Deshpande V, Zhu AX. Genetics of biliary tract cancers and emerging targeted therapies. J Clin Oncol 2010;28:3531-3540.

48 Deshpande V, Nduaguba A, Zimmerman SM, et al. Mutational profiling reveals PIK3CA mutations in gallbladder carcinoma. BMC Cancer 2011;11:60.

49 Levi S, Urbano-Ispizua A, Gill R, et al. Multiple K-ras codon 12 mutations in cholangiocarcinomas demonstrated with a sensitive polymerase chain reaction technique. Cancer Res 1991;51:3497-3502.

50 Wood LD, Hruban RH. Pathology and molecular genetics of pancreatic neoplasms. Cancer J 2012;18: 492-501.

51 Ottenhof NA, de Wilde RF, Maitra A, et al. Molecular characteristics of pancreatic ductal adenocarcinoma. Patholog Res Int 2011;2011:620601.

52 Hezel AF, Kimmelman AC, Stanger BZ, et al. Genetics and biology of pancreatic ductal adenocarcinoma. Genes Dev 2006;20:1218-1249.

53 Schultz NA, Roslind A, Christensen IJ, et al. Frequencies and prognostic role of KRAS and BRAF mutations in patients with localized pancreatic and ampullary adenocarcinomas. Pancreas 2012;41:759-766.

54 Schonleben F, Qiu W, Allendorf JD, et al. Molecular analysis of PIK3CA, BRAF, and RAS oncogenes in periampullary and ampullary adenomas and carcinomas. J Gastrointest Surg 2009;13:1510-1516.

55 Hoang MP, Murakata LA, Katabi N, et al. Invasive papillary carcinomas of the extrahepatic bile ducts: a clinicopathologic and immunohistochemical study of 13 cases. Mod Pathol 2002;15:1251-1258.

56 Phipps AI, Buchanan DD, Makar KW, et al. BRAF mutation status and survival after colorectal cancer diagnosis according to patient and tumor characteristics. Cancer Epidemiol Biomarkers Prev 2012;21: 1792-1798.

57 Dienstmann R, Serpico D, Rodon J, et al. Molecular profiling of patients with colorectal cancer and matched targeted therapy in phase I clinical trials. Mol Cancer Ther 2012;11:2062-2071.

58 Prahallad A, Sun C, Huang S, et al. Unresponsiveness of colon cancer to BRAF(V600E) inhibition through feedback activation of EGFR. Nature 2012;483: 100-103. 